\title{
Erratum: Association between Salivary Fructosamine, Plasma Glycated Hemoglobin, and Plasma Glucose Levels among Type II Diabetes Mellitus and Nondiabetic Individuals-A Cross-sectional Study
}

Sadhana Kandavel ${ }^{1} \quad$ P. D. Madan Kumar ${ }^{2}$

\footnotetext{
${ }^{1}$ Department of Public Health Dentistry, Sree Balaji Dental College and Hospital, Chennai Tamil Nadu, India

2Department of Public Health Dentistry, Ragas Dental College and Hospital, Chennai, Tamil Nadu, India
}

Address for correspondence Sadhana Kandavel, Department of

Eur J Dent 2019;00:e1

ERRATUM

It has been brought to the Publisher's attention that the affiliations were appearing incorrectly in the above article in the European Journal of Dentistry, published online on June 28, 2019 (DOI: 10.1055/s-0039-1692018). The authors' correct affiliations appear as above. 